\title{
MR imaging of the reconstructed breast: What the radiologist needs to know
}

\author{
Vandana Dialani • Kenny C. Lai • Priscilla J. Slanetz
}

Received: 14 January 2012 / Accepted: 23 January 2012 / Published online: 17 March 2012

(C) European Society of Radiology 2012

\begin{abstract}
Objective The objective is to review the different types of breast reconstruction following cancer surgery and describe expected imaging appearances and complications seen in the reconstructed breast.

Methods Surgical management of breast cancer often entails lumpectomy or mastectomy. When mastectomy is performed, patients often opt for breast reconstruction. Most facilities do not routinely image the reconstructed breast with mammography.

Results However, many of these women are imaged with screening breast MRI for evaluation of the contralateral breast, or they may develop a clinical problem that warrants a diagnostic evaluation with MRI. In this article, we will review the more commonly encountered types of breast reconstruction, which include implants, tranversus rectus abdomnis flap, latissimus dorsi flap, deep inferior epigastric perforator flap, and gluteal flaps. Each of these types of reconstruction has different appearances on MR. We will also discuss potential complications that can be seen in the reconstructed breasts, including fat necrosis and recurrence. Conclusion Radiologists will better understand the different types of breast reconstruction after mastectomy and their normal imaging appearance on MRI. Radiologists will be more aware of how to recognize complications related to surgery as well as how to determine whether recurrence is present.
\end{abstract}

V. Dialani $(\varangle) \cdot$ K. C. Lai $\cdot$ P. J. Slanetz

Department of Radiology, Beth Israel Deaconess Medical Center,

C/o Dr. Vandana Dialani, 330 Brookline Avenue,

Boston, MA 02215, USA

e-mail: vdialani@bidmc.harvard.edu
Main Messages

- The different surgical techniques used in breast reconstruction are discussed.

- Describes the normal magnetic resonance imaging appearance of the breast after reconstruction.

- Identify MR imaging features of benign sequelae and recurrence following breast reconstruction.

Keywords Breast · MRI imaging · Surgery · Types of breast reconstruction $\cdot$ MRI imaging of reconstructed breast

\section{Introduction}

Surgical management of breast cancer often entails lumpectomy or mastectomy, followed usually by breast reconstruction. Each of these types of reconstruction has different appearances on magnetic resonance imaging (MRI) examinations. Understanding the expected anatomic appearance of the breast subsequent to breast reconstruction is critical in the interpretation of MRI following surgery. It is also important to be aware of the potential complications seen in the reconstructed breast and their MRI appearances.

\section{MRI acquisition}

In our institution, breast MRI is performed at 1.5-T (GE Healthcare, Milwaukee, WI, USA) to produce high-spatialresolution images in the axial plane. Unenhanced fat-saturated T1-weighted (T1W) images are obtained followed by contrastenhanced acquisition of fat-saturated T1W images with data at three time points, in consecutive 3- to 5-min intervals. Delayed sagittal T1W fat-saturated images are also obtained. In addition, $\mathrm{T} 2 \mathrm{~W}$ nonfat-saturated images are obtained, which gives 
architectural information. The contrast agent gadopentetate dime-glumine (Magnevist, Bayer Schering Pharma) was administered intravenously at a dose of $0.1 \mathrm{mmol} / \mathrm{kg}$ body weight with an MRI-compatible remote control power injector at a rate of $1.2 \mathrm{ml} / \mathrm{s}$ in all studied patients. The contrast injection was followed by a $10-\mathrm{ml}$ saline flush administered at the same flow rate. The imaging parameters were as follows: TR/TE, 9/4.4; flip angle, $10^{\circ}$; number of signals acquired, 1; acquisition matrix, $512 \times 512$; section thickness, $2 \mathrm{~mm}$.

Common problems in MRI of reconstructed breasts are failure of fat suppression and inhomogeneous fat suppression. Field homogeneity can be improved by adjustment of the shim gradients and ensuring that shim volumes adequately cover the breast tissue. In addition, the center frequency should be adjusted to the center of the water peak for optimal fat suppression. If fat suppression remains a problem, images can be obtained without fat suppression while the patient is instructed to remain very still. Subtraction images can then be used for interpretation.

Given that there are few articles on this topic in the radiology literature, we studied the patients with breast reconstructions who had an MRI at our institution to understand different types of reconstructions performed by the surgeons and the encountered complications.

\section{Different surgical techniques used in breast reconstruction}

Various reconstruction techniques are described in Table 1. Based on individual factors such as general health, lifestyle, emotional state as well as breast size and shape, the choice of reconstruction can be made. The indications, contraindications, disadvantages, and advantages are tabulated in Table 2. It is important to understand the surgical procedure to identify

Table 1 Different surgical techniques used in breast reconstruction

\begin{tabular}{|c|c|}
\hline $\begin{array}{l}\text { Expander/implant } \\
\text { reconstruction }\end{array}$ & Autologous flap reconstruction \\
\hline $\begin{array}{l}+/- \text { Acellular matrix } \\
\text { (AlloDerm, Allomax, etc.) }\end{array}$ & (A) Pedicled \\
\hline \multirow[t]{6}{*}{$\begin{array}{l}+/- \text { Latissimus muscle } \\
\text { flap with implant }\end{array}$} & $\begin{array}{l}\text { Transplantation of a transverse rectus } \\
\text { abdominis myocutaneous (TRAM) }\end{array}$ \\
\hline & $\begin{array}{l}\text { Reconstruction with the latissimus } \\
\text { dorsi myocutaneous flap }\end{array}$ \\
\hline & (B) Free flap \\
\hline & $\begin{array}{l}\text { DIEP/SIEA (Deep inferior epigastric } \\
\text { artery perforator flap/superficial } \\
\text { inferior epigastric artery flap) }\end{array}$ \\
\hline & $\begin{array}{l}\text { SGAP/IGAP (Superior gluteal artery } \\
\text { perforator flap or inferior gluteal } \\
\text { artery perforator flap) }\end{array}$ \\
\hline & TUG (Transverse upper gracilis flap) \\
\hline
\end{tabular}

the MRI appearance of the reconstructed breast; hence, effort has been made to explain each surgical procedure briefly, which helps understand the normal MRI appearance.

\section{Expander/implant reconstruction}

Postmastectomy reconstruction with a tissue expander and implant involves a staged approach. A tissue expander is a temporary device placed on the chest wall deep to the pectoralis major muscle, which may be performed immediately following the mastectomy or as a delayed procedure. The purpose is to create a soft pocket to contain the permanent implant. Tissue expanders are available in a variety of shapes and sizes. Certain tissue expanders have a magnet filling port and are not MRI compatible. When it is first positioned, it is partially filled with saline. Within a few weeks, once the patient has healed, expansion is started as an outpatient procedure at 1-, 2-, or 3-week intervals over several months. The timing of expansion can be coordinated with chemotherapy treatments.

Once expansion is completed and the patient is medically cleared for another operation (about 1 month after chemotherapy), the second stage of reconstruction is performed. This is an outpatient procedure that involves exchanging the expander for an implant and creating a more refined breast shape. The initial tissue expander placement and the subsequent exchange for an implant each take about $1 \mathrm{~h}$ in the operating room. Implant-based reconstruction may be combined with acellular dermal matrix coverage or with a lattisimus dorsi flap.

\section{Advantages of acellular dermal matrix}

The use of an acellular dermal matrix such as AlloDerm allows the surgeon to place a higher volume of saline during the initial surgery, which decreases the number of expansions needed later, and the patient could have an immediate breast shape after the first operation (Fig. 1a). Often, the only available coverage for implant-based reconstruction is a thin layer of skin and subcutaneous tissue, which predisposes to the formation of capsular contracture, producing an unnaturally round breast mound. Breast implants covered with acellular dermal matrix (AlloDerm) (Fig. 1b, c) are less likely to develop a capsule in the area where the implant is in direct contact with the acellular matrix [1].

\section{Normal MR imaging appearance of breast implant covered with acellular matrix}

AlloDerm is isointense to glandular parenchyma on T1W images and does not enhance with gadolinium. It will be 


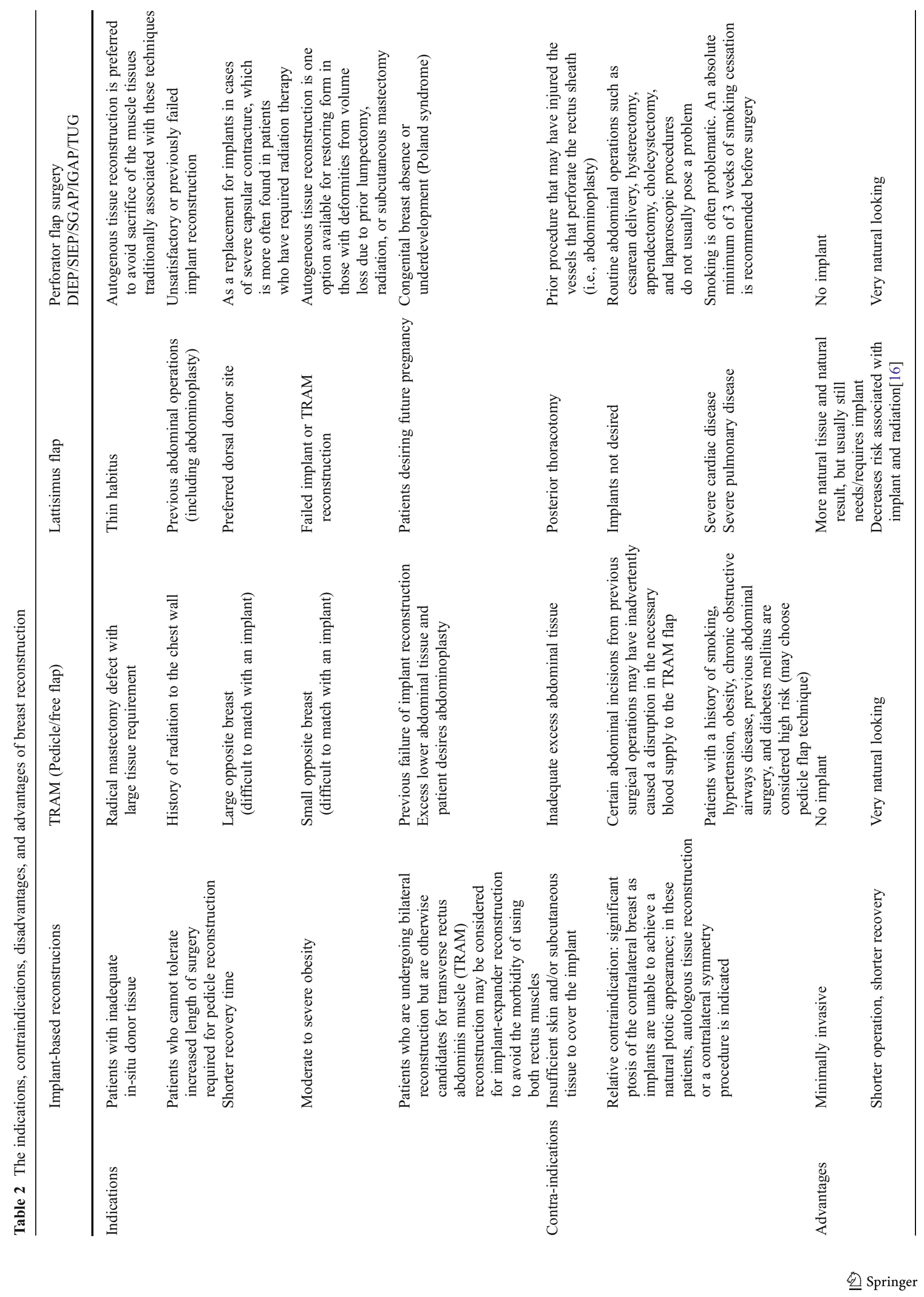




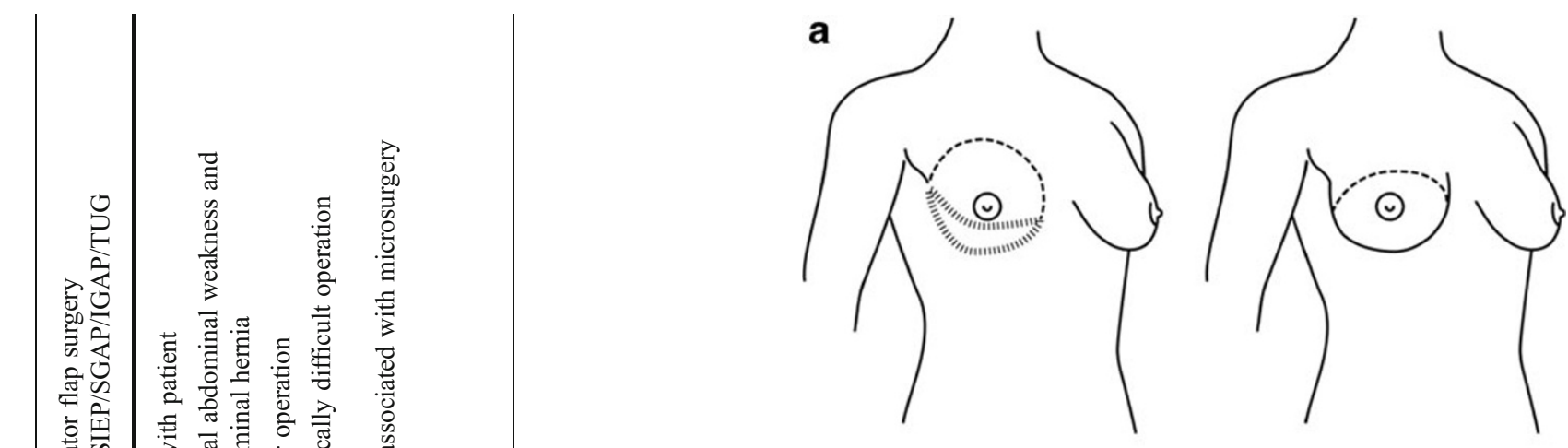

b

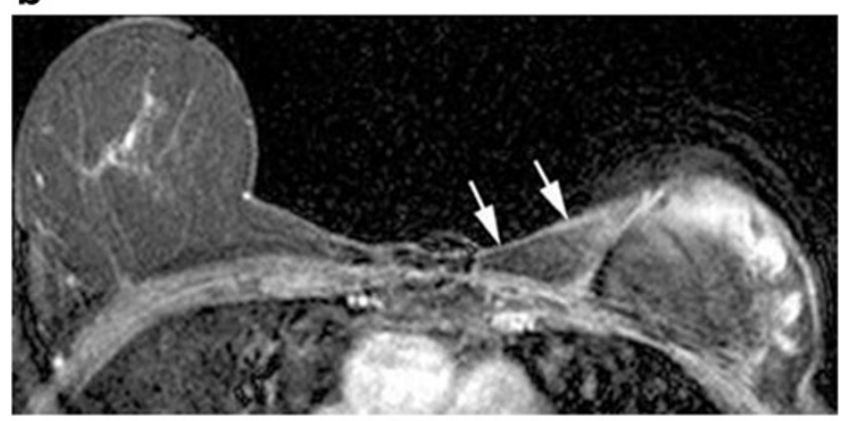

c

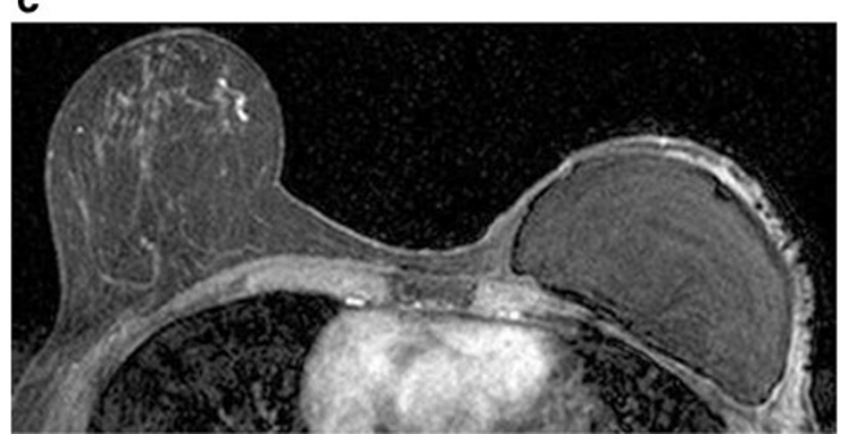

Fig. 1 a Schematic showing use of AlloDerm for implant reconstruction. The dotted lines represent the AlloDerm used for reconstruction, which contains the implant. b, c) Axial T1W fat-saturated contrastenhanced images, showing AlloDerm used for breast reconstruction seen medially (arrows) and inferior image showing the saline implant in place with overlying skin thickening

seen overlying the implant, filling the gap between the skin and the implant (Figs. 1b, c). The figures help identify the normal appearance of AlloDerm.

\section{Reconstruction with the latissimus dorsi myocutaneous flap}

The latissimus dorsi (LD) flap is a standard method for breast reconstruction that was first utilized in the 1970s. It is most commonly combined with a tissue expander or implant, or occasionally, for a thin patient with a small breast volume, can be used alone as the primary reconstruction without the need for an implant, restoring volumes of 
Fig. 2 a Reconstruction with latissimus dorsi myocutaneous flap. Latissimus dorsi muscle, fat, and skin are rotated to reconstruct the breast. b? Axial fat-saturated T1W image with post-contrast gadolinium injection, the flap consisting of the latissimus dorsi muscle and its overlying skin and fat flipped and tunneled from the back to the neobreast (white arrows), giving a tailed appearance to the muscle in the lateral breast. This can be used as a differentiator on imaging from TRAM flap reconstruction. c Sagittal nonfat-saturated T2W image show latissimus dorsi muscle flipped anteriorly for reconstruction, and the denuded dermal layer is seen parallel to the chest wall (white arrow heads)

up to 1.51 in large patients or with the use of modified techniques [2, 3], which is considered oncologically safe. It is often reserved for patients in whom TRAM reconstruction is contraindicated. It can also be used as a salvage procedure for patients who have had previous radiation and are not candidates for other autogeneous procedures. Reconstruction with the latissimus dorsi myocutaneous flap produces a breast with ptosis and projection while maintaining the natural consistency and feel of normal tissue (Fig. 2).

\section{Normal MR imaging appearance of latissimus dorsi myocutaneous flap}

On the MR images, the latissimus dorsi muscle flipped inferiorly for reconstruction is well appreciated. The flap consists of the latissimus dorsi muscle and its overlying skin and fat flipped and tunneled from the back to and from the neobreast, giving a tailed appearance to the muscle in the lateral breast. This can be used as a differentiator on imaging from TRAM flap reconstruction. This atrophies over time, showing fatty strands within. The denuded dermal layer is seen parallel to the skin of the breast (Fig. 2).

\section{Reconstruction with the TRAM (pedicle/free flap)}

Transplantation of a TRAM flap is a commonly used surgical procedure for breast reconstruction following mastectomy. Since the description of this procedure by Hartrampf et al. [4] in 1982, numerous refinements of the basic technique have been developed, which include the pedicled, free, and delayed flap reconstruction. In the TRAM flap technique, the rectus abdominis muscle must be harvested along with the subcutaneous soft tissue to ensure the preservation of the perforating vessels that supply the abdominal skin and subcutaneous fat. The rectus abdominis muscle has a dual blood supply, which includes the superior and inferior epigastric vessels. The two major technical variants of the TRAM flap procedure include the pedicled flap (Fig. 3) and microsurgical free flap reconstructions.

The pedicled TRAM flap is based on the superior epigastric vasculature and requires the full length of the rectus abdominis muscle. The harvested muscle and lower abdominal

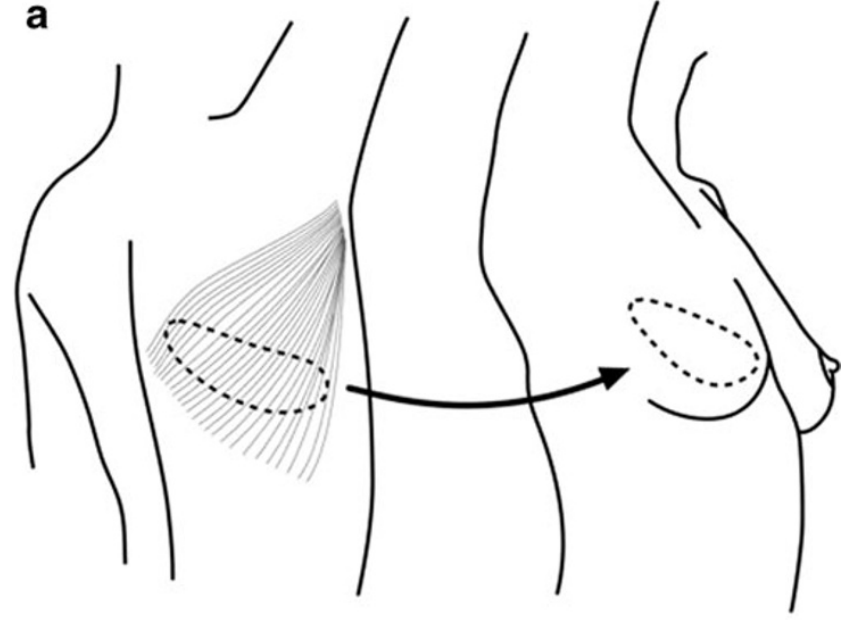

b

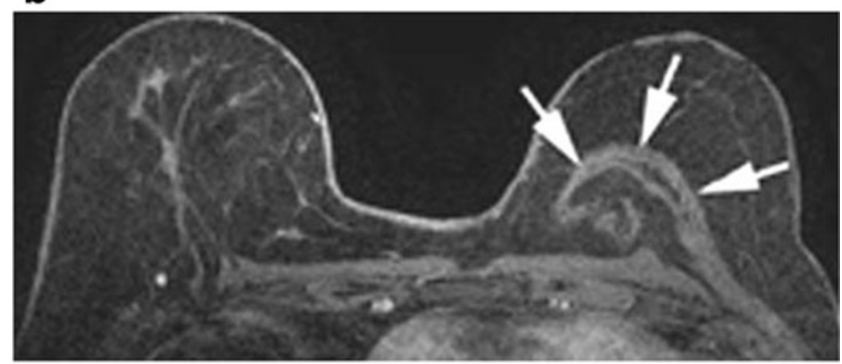

c

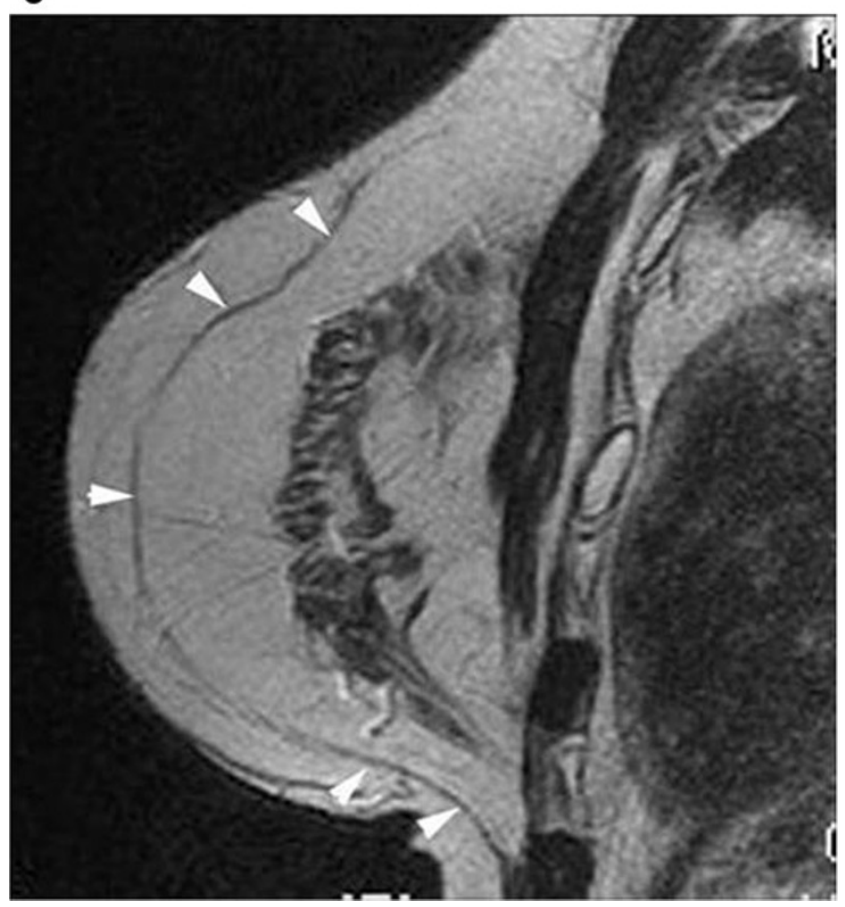

soft tissue are elevated and tunneled subcutaneously into the mastectomy defect. The portion of the abdominal epidermis that is used to create the skin surface of the neobreast remains intact, whereas the remainder is de-epithelialized. The contralateral rectus abdominis muscle is more commonly used for 
Fig. 3 a Pedicled TRAM flaprectus abdominis muscle rotated up to the chest for breast reconstruction. b Sagittal fat-saturated T1W image with post-contrast gadolinium injection shows replacement of the normal glandular tissue of the breast with lower abdominal fat and the presence of atrophied rectus abdominis muscle along the anterior chest wall (black arrow). c Axial fat-saturated T1W images with dynamic gadolinium injection showing the atrophied rectus abdominis muscle along the anterior chest wall (arrow) and the bulk of the muscle in the center as opposed to the eccentric location in the latissimus flap. The lower abdominal fat constitutes the right reconstructed breast. Normal left breast for comparison

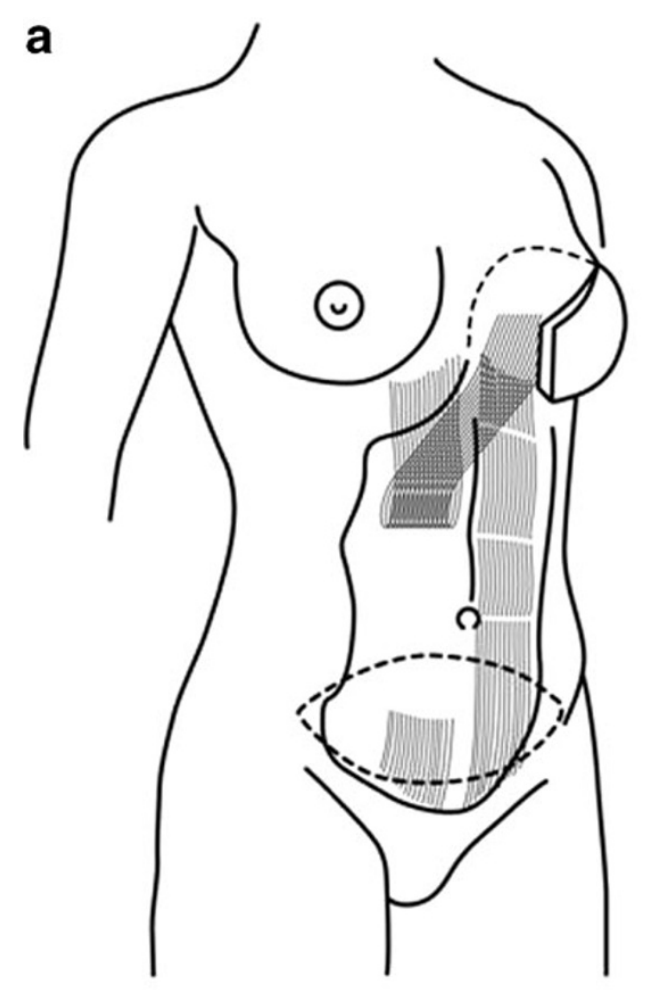

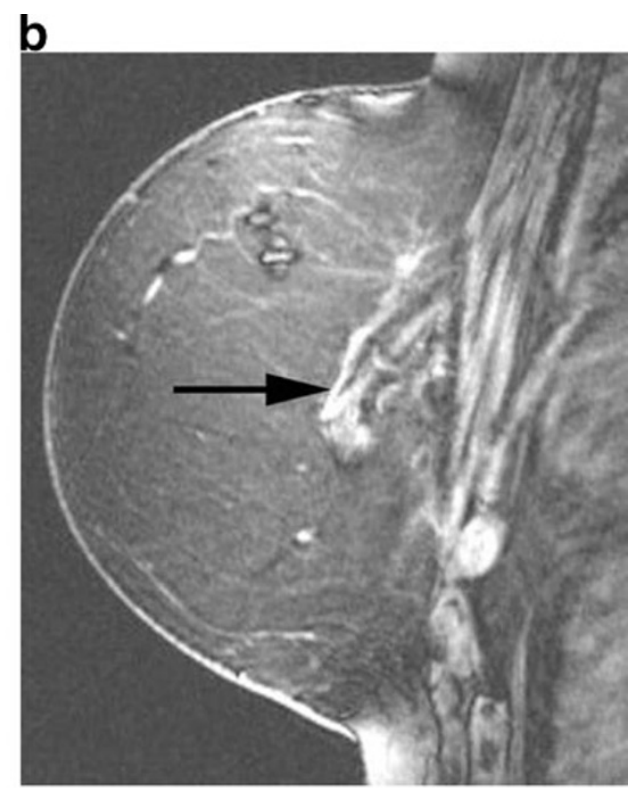

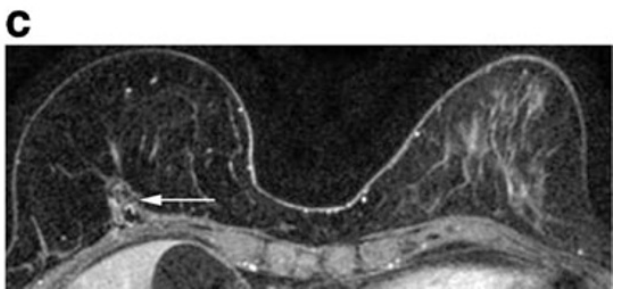

unilateral reconstruction. When bilateral reconstruction is performed, the ipsilateral rectus abdominis muscle is used in order to prevent crossing of the pedicles and subsequent potential vascular compromise.

The free $\boldsymbol{T R} \boldsymbol{A} \boldsymbol{M}$ flap derives its blood supply from the inferior epigastric artery and vein. The inferior epigastric is chosen, as the blood supply via the superior epigastric artery is attenuated along its course by multiple tendinous inscriptions and is less robust. These vessels are ligated and microsurgically re-anastomosed in the chest to the thoracodorsal, subscapular, or internal mammary artery and vein. The microsurgical technique is preferred in patients with relative risk factors for atherosclerosis including obesity, diabetes mellitus, smoking, and cardiovascular disease, as well as in those patients who have undergone prior abdominal surgery [5]. Compared with the pedicled flap, the free flap requires a smaller amount of rectus abdominis muscle, resulting in decreased prevalence of abdominal wall hernia.

\section{Normal MR imaging appearance of transplantation of a transverse rectus abdominis myocutaneous (TRAM) flap}

The most familiar changes of TRAM flap reconstruction include the replacement of the normal glandular tissue of the breast with lower abdominal fat and the presence of atrophied rectus abdominis muscle along the anterior chest wall (Fig. 3). Note in TRAM reconstruction the bulk of the flap is usually in the center on the MRI image especially on the axial images, as oppposed to the latissimus flaps where the flap consisting of the latissimus dorsi muscle and its overlying skin and fat is rotated and tunneled from the back to the neobreast, giving a tailed appearance to the muscle in the lateral breast. This can be used as a differentiator on imaging.

\section{Free flap reconstruction: (DIEP/SIEA; SGAP/IGAP; TUG)}

Perforator flaps allow the transfer of the patient's own skin and fat in a reliable manner with minimal donor-site morbidity. During flap harvest, these perforators are meticulously dissected free from the surrounding muscle, which is spread in the direction of the muscle fibers and preserved intact. The pedicle is anastomosed to recipient vessels in the chest, and the donor site is closed without the use of mesh or other materials [6-8] (Figs. 4, 5). The flap ages with the patient, and the perforator flaps preserve natural function of muscles. The procedure involves microsurgical techniques; hence, it is tolerated longer and better by young, healthy patients. 
Fig. 4 a The DIEP flap is supplied by intramuscular perforators from the deep inferior epigastric artery and vein. $\mathbf{b}$ Axial fat-saturated T1W images with post-contrast gadolinium injection. c 3D reformatted axial image show replacement of the normal glandular tissue of the breast with lower abdominal fat and the anastomosis of the vascular pedicle (arrow) by microsurgical technique to the internal mammary artery. For comparison, note the normal glandular tissue in the left breast

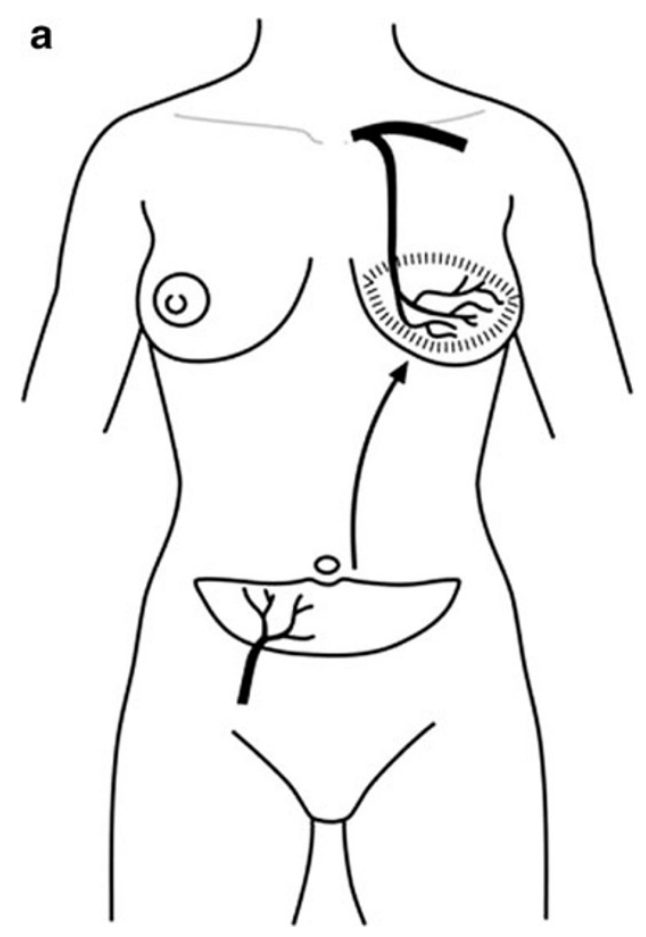

b
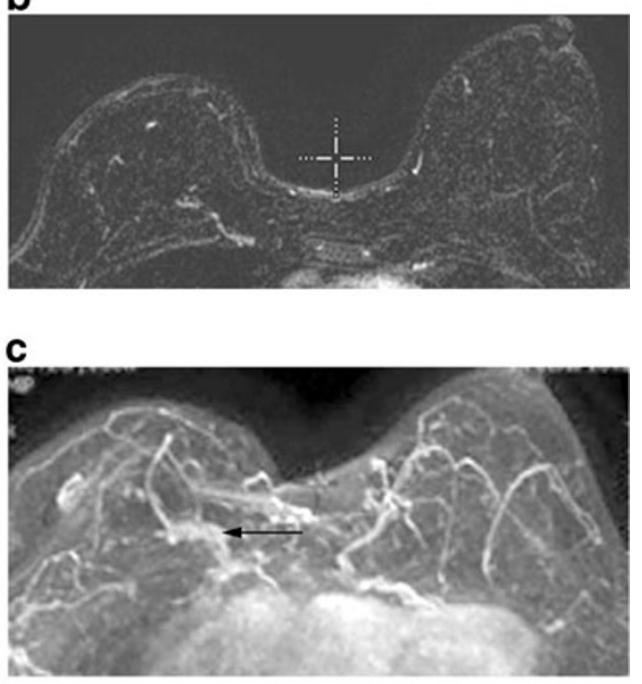

The Deep inferior epigastric artery perforator (DIEP) and superficial inferior epigastric artery (SIEA) flaps transfer the same tissue from the abdomen to the chest for breast reconstruction as the TRAM flap without sacrificing the rectus muscle or fascia. The DIEP flap is supplied by intramuscular perforators from the deep inferior epigastric artery and vein. The SIEA flap is based on the SIEA and vein, which arise from the common femoral artery and saphenous bulb.

The GAP flaps are based on perforators from either the superior or inferior gluteal artery (Fig. 5). The superior gluteal artery perforator (S-GAP) flap is the preferred method of breast reconstruction when the abdomen is not available or preferable [9]. Gluteal artery perforator (GAP) flaps allow transfer of tissue from the buttock, also with minimal donor-site morbidity.

The transversus upper gracilis (TUG) flap procedure uses tissue from the inner portion of the upper thigh (just under the groin crease) for breast reconstruction (Fig. 5). Loss of the gracilis muscle does not result in any noticeable functional impairment. The ideal shape of the TUG flap allows shaping of the breast with a very aesthetic contour and projection.

\section{Normal MR imaging appearance of free flap reconstruction}

Free flap reconstruction on MRI shows replacement of the normal glandular tissue of the breast with tissue from the lower abdomen, buttocks, or thigh fat. It also shows a thin vascular pedicle that is anastomosed by microsurgical technique to the internal mammary artery (Fig. 4,5 ).
All free flap reconstructions show the reconstructed breast constituted of fat and the vascular pedicle, which is the anastomosis site to the internal mammary artery. There is no muscle seen in these as opposed to the TRAM or latissimus reconstruction, which has atrophied muscle along the chest wall.

\section{MR imaging of the postoperative breast}

In the postoperative breast, radiation-induced changes, including fibrosis and skin thickening complicate clinical evaluation, and distortion of normal breast architecture confounds physical examination and mammographic assessment of the breast. MR imaging has been demonstrated to be extremely useful for demonstrating clinically occult breast cancer, identifying unsuspected multifocal cancer and differentiating benign postoperative findings from recurrent breast cancer. The specificity of MR imaging in the irradiated breast may vary, with more prominent enhancement present for up to 1 year following radiation treatment, and enhancement may be present for several years.

\section{Benign sequalae after flap reconstruction}

Seromas and hematomas may be seen after breast reconstruction surgery. Seromas may demonstrate unilocular or complex $\mathrm{T} 2$ bright signal. Hematomas show variable signal intensity on $\mathrm{T} 1$ - and $\mathrm{T} 2$-weighted images depending on the evolution of the blood products. In addition, they often demonstrate a low- 


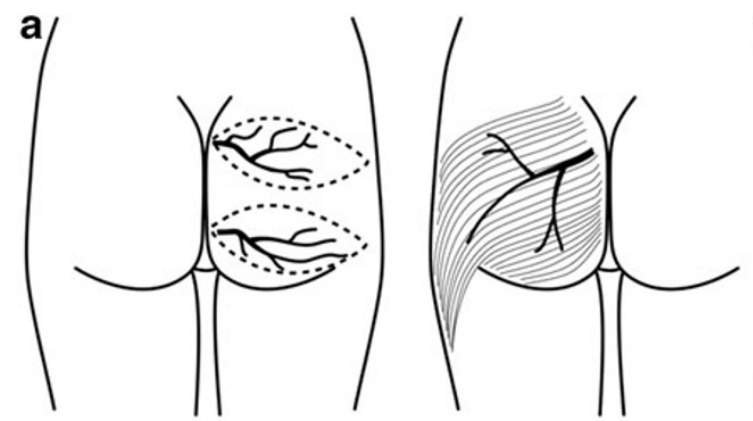

b

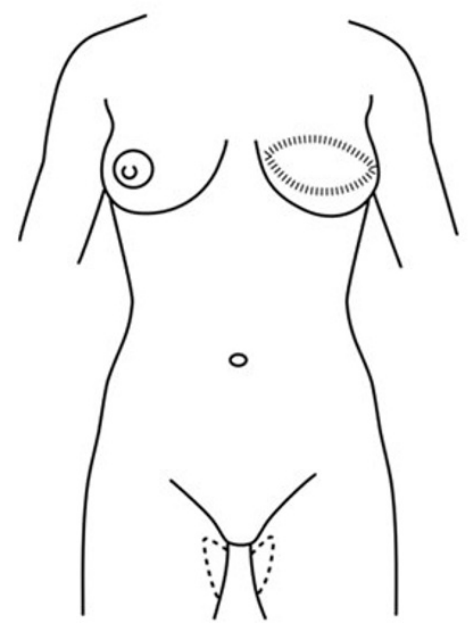

Fig. 5 a GAP flaps are based on perforators from either the superior or inferior gluteal artery. Superior gluteal artery perforator (SGAP) flaps allow transfer of tissue from the buttock using the superior gluteal arty as a vascular pedicle for transfer of tissue. Inferior gluteal artery perforator (IGAP) flaps allow transfer of tissue from the buttock using the inferior gluteal artery as a vascular pedicle for transfer of tissue. b The transversus upper gracilis (TUG) flap procedure uses tissue from the inner portion of the upper thigh for breast reconstruction. c Axial

signal-intensity rim of hemosiderin. Uniform rim enhancement may be seen, but there should be no solid or internal enhancement within a postoperative seroma or hematoma.

\section{Skin thickening}

Radiation therapy causes vasodilatation and hinders venous outflow within the skin and subcutaneous tissues. Reconstruction may affect venous and lymphatic drainage, contributing further to fluid accumulation, especially with TRAM flap reconstruction [10]. On MR imaging, benign skin thickening can be identified as a diffuse band of tissue that is dark on T1-weighted images and bright on T2weighted images, and usually does not show enhancement (Fig. 6). These findings are nonspecific, and tumor infiltration in inflammatory carcinoma can manifest similarly. Skin
C

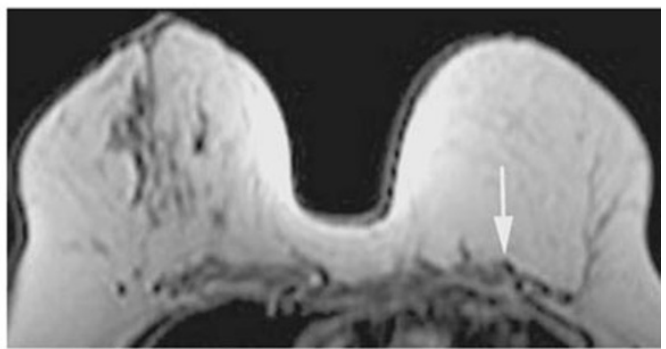

d

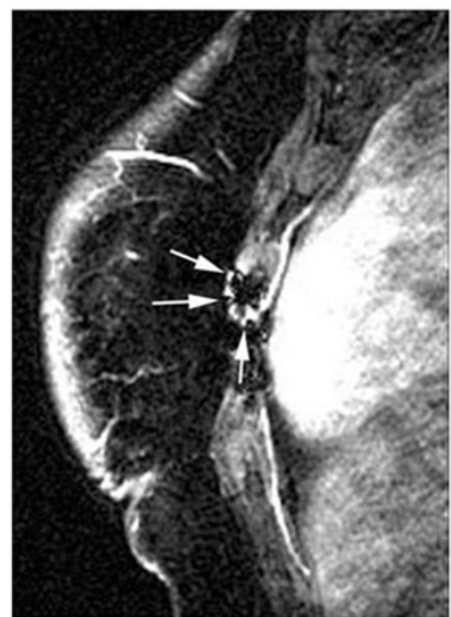

nonfat-saturated T2W axial image showing, SGAP reconstruction with microvascular surgical anastomosis of the superior gluteal artery to the internal mammary artery (white arrow). For comparison, note the normal glandular tissue in the right breast. d Sagittal fat-saturated T1W image with dynamic gadolinium injection shows replacement of the normal glandular tissue of the breast with lower abdominal fat and the anastomosis of the vascular pedicle by microsurgical technique to the internal mammary artery (white arrow)

enhancement following radiation therapy is more uniform and less intense. Inflammatory cancer, however, has more

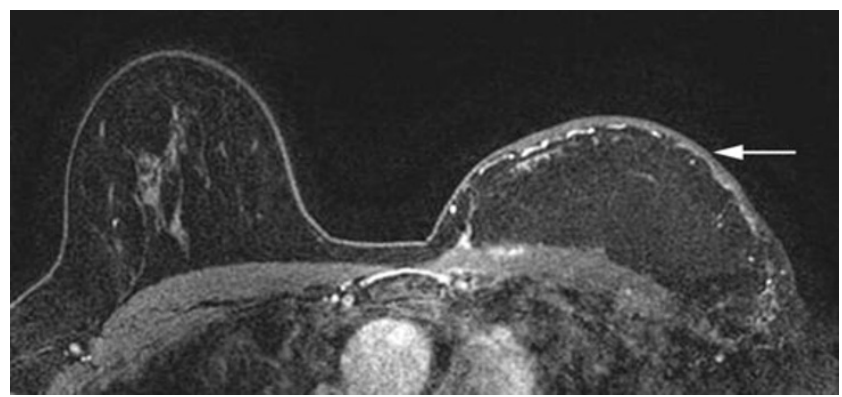

Fig. 6 Benign skin thickening post-TRAM flap breast reconstruction identified as a diffuse band of tissue that is bright on T1W unenhanced image, and as shown here no significant enhancement is seen on postcontrast T1W image. Note enhancement in the underlying vessels 


\section{a}

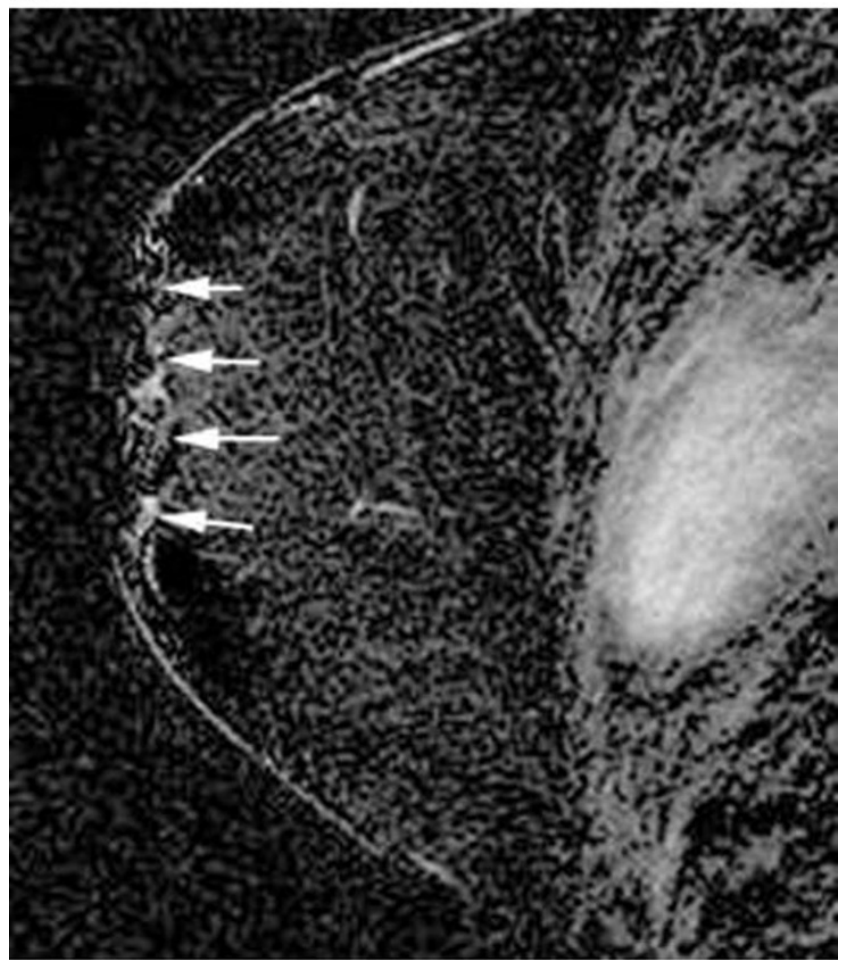

b

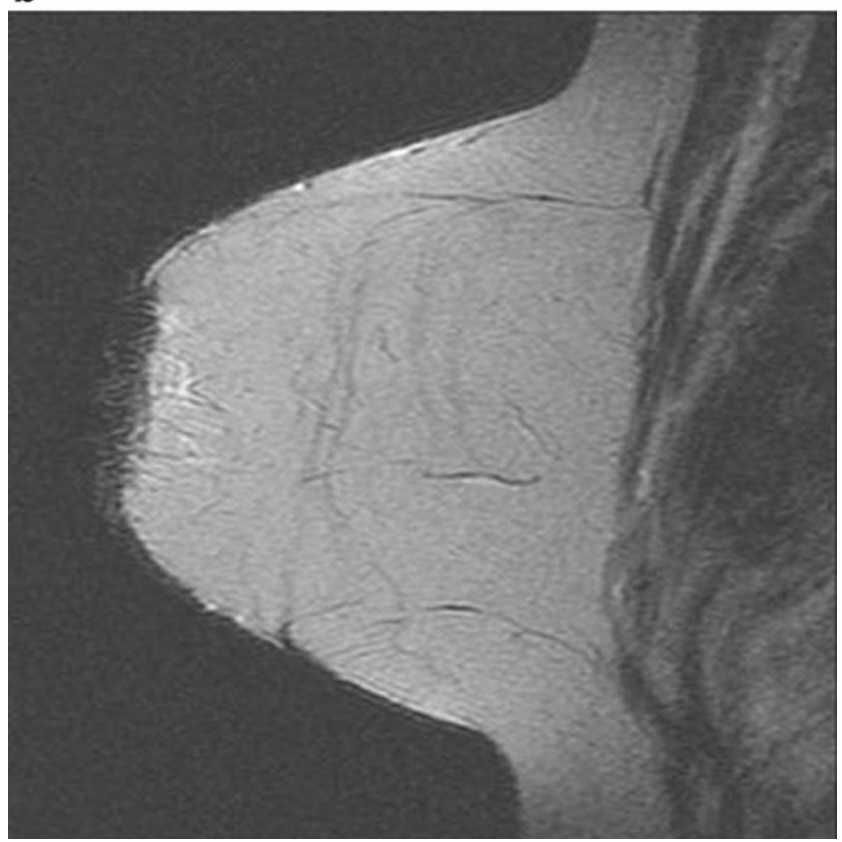

Fig. 7 A 57-year-old woman with TRAM flap reconstruction and nipple tattoo: a $\mathrm{T} 1 \mathrm{~W}$ fat-saturated contrast-enhanced and b) $\mathrm{T} 2 \mathrm{~W}$ nonfat-saturated images showing as an area of signal void along the nipple areola complex (arrows) because of the pigments used for nipple tattooing

marked skin enhancement, with underlying tumor enhancement in either a septal or solid pattern [11].

\section{Nipple tattoo artifact after flap reconstruction}

Nipple-areola reconstruction represents the completion of the breast restorative process and has significant psychological implications for women who undergo mastectomy. Complete nipple-areola reconstruction with tattoos can visually draw attention away from the scars on the reconstructed breast mound. The base powder, initially barium sulfate, was later replaced by titanium dioxide. Masser et al. introduced the pigment-gel-suspension technique in 1989, achieving more natural results by avoiding a painted appearance. The fineparticle pigments, when introduced into the dermis, resemble the distribution of natural skin pigments, such as melanin in melanocytes and hemoglobin in skin capillaries [12]. On MR

\section{a}

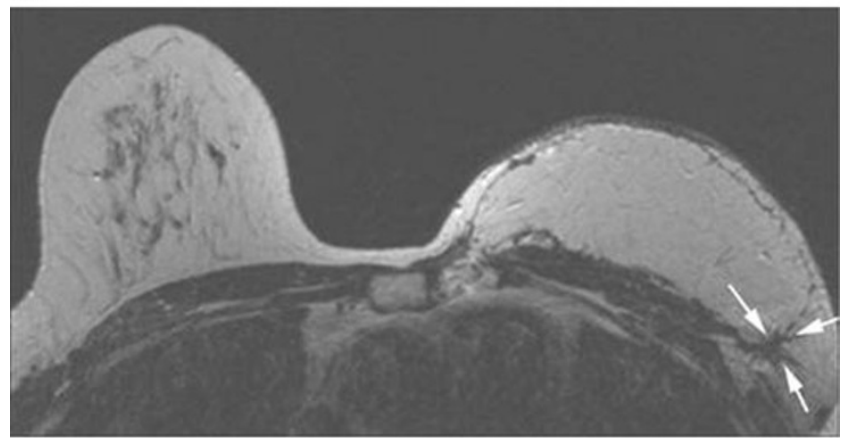

b

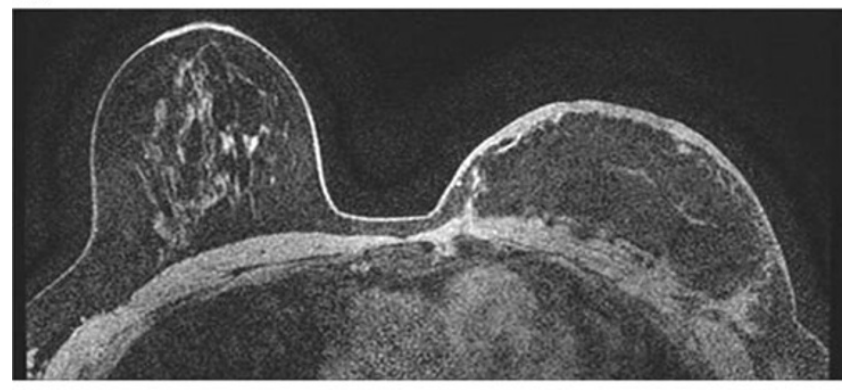

C

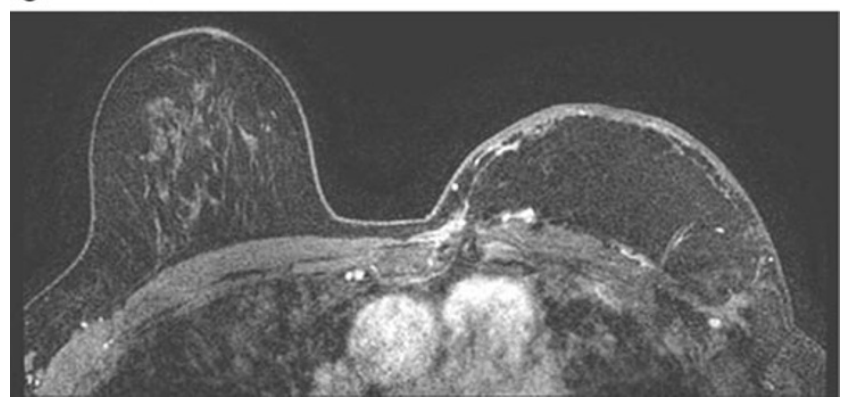

Fig. 8 Image of a 58-year-old woman with DIEP reconstruction of the left breast showing a spiculated area (arrow) in the posterior breast. The mass demonstrates a low signal intensity on the T2W image, b intermediate signal intensity on the $\mathrm{T} 1 \mathrm{~W}$ fat-saturated unenhanced image, and $\mathbf{c}$ no enhancement on the gadolinium-enhanced $\mathrm{T} 1 \mathrm{~W}$ image, indicative of fibrosis 
a

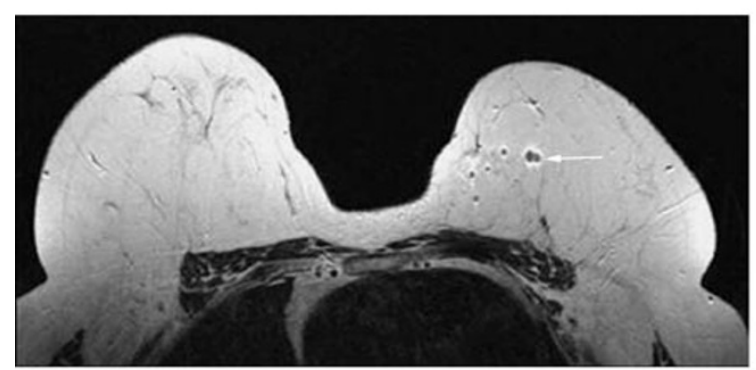

C

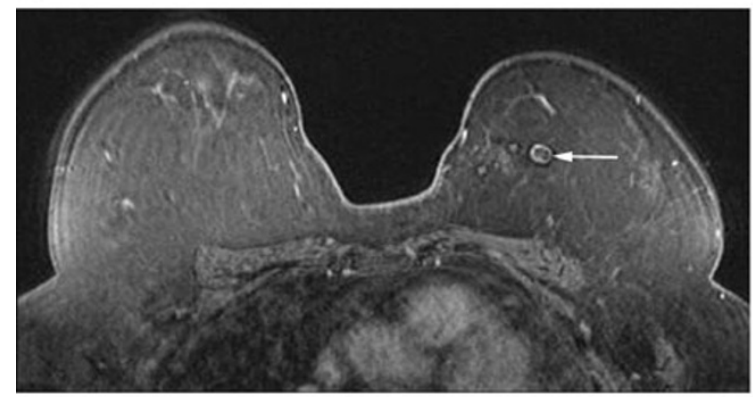

e

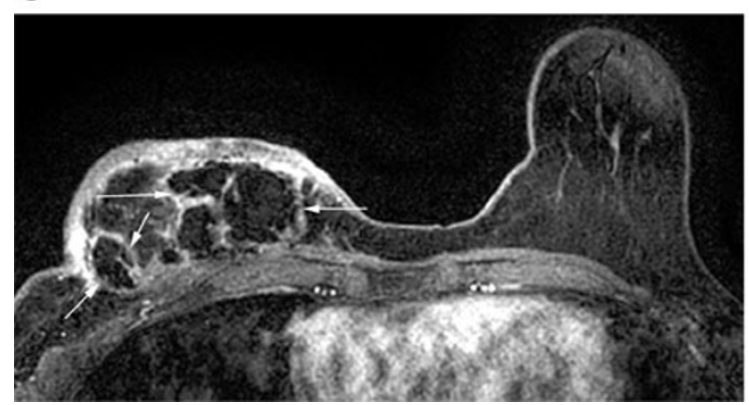

b

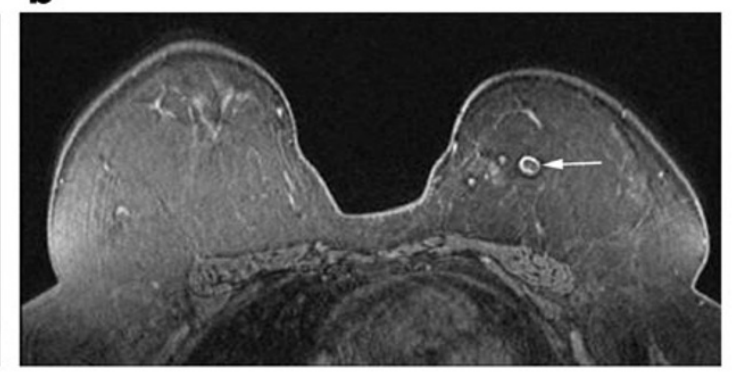

d

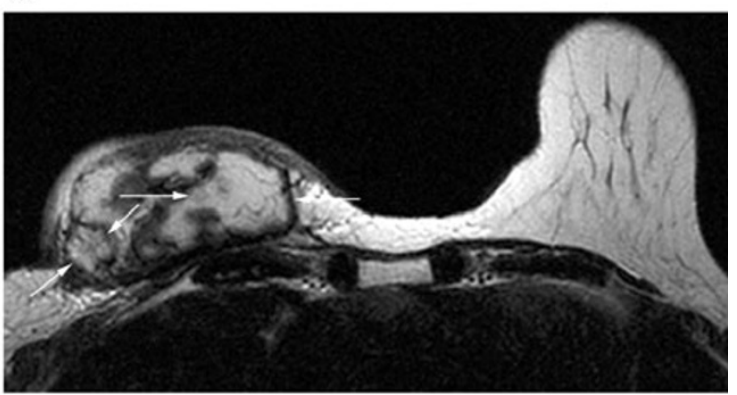

Fig. 9 A 54-year-old woman with history of left breast cancer treated with mastectomy and TRAM reconstruction 10 years prior. a T2W, b unenhanced T1W fat saturated. $\mathbf{c}$ Enhanced T1W fat-saturated sequences demonstrate post-treatment changes within the left TRAM demonstrating minimal peripheral enhancement, which follows the fat signal on all sequences (arrows), consistent with fat necrosis. d Another

imaging the tattoo produces a blooming artifact on the $\mathrm{T} 1$ and $\mathrm{T} 2 \mathrm{~W}$ images with a faint rim of discontinuous enhancement on the subtraction images (Fig. 7).

\section{Benign complications}

\section{Fibrosis}

Fibrosis is a common sequel of radiation therapy in the breast. Atrophy and fibrosis of the transplanted muscle are also seen as a sequel to reconstructed breast. One of the challenges in evaluating the postoperative breast is to differentiate between postoperative or post-radiation therapy scarring and tumor recurrence. Here MR imaging may often prove helpful, as post-radiation therapy fibrosis often displays low signal patient, 53 years old, with DIEP reconstruction on the right presented with palpable lumps on the right, 2 years post-reconstruction surgery. Unenhanced $\mathrm{T} 2 \mathrm{~W}$ and enhanced T1W fat-saturated images demonstrate multiple masses (arrows) in the left breast showing a fat signal within the masses and minimal peripheral enhancement, consistent with fat necrosis

intensity on T2-weighted images, whereas tumor recurrence is generally isointense to slightly hyperintense to the surrounding

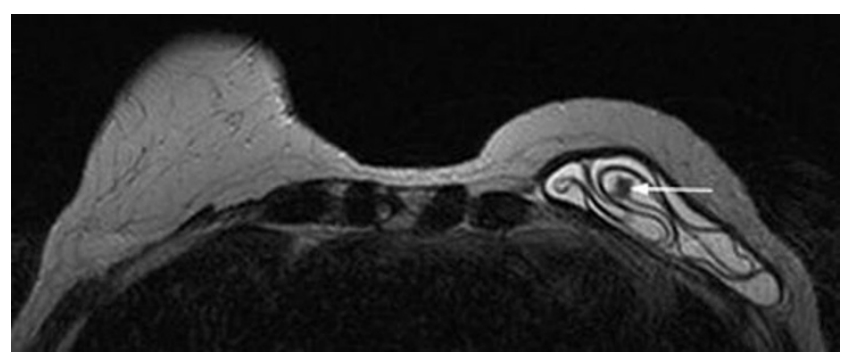

Fig. 10 A 66-year-old women with a history of left breast cancer treated with mastectomy in 1990, with saline implant reconstruction. Axial T2W images demonstrate the intracapsular rupture of this saline implant. The valve of the deflated implant is seen on the image (white arrow) 


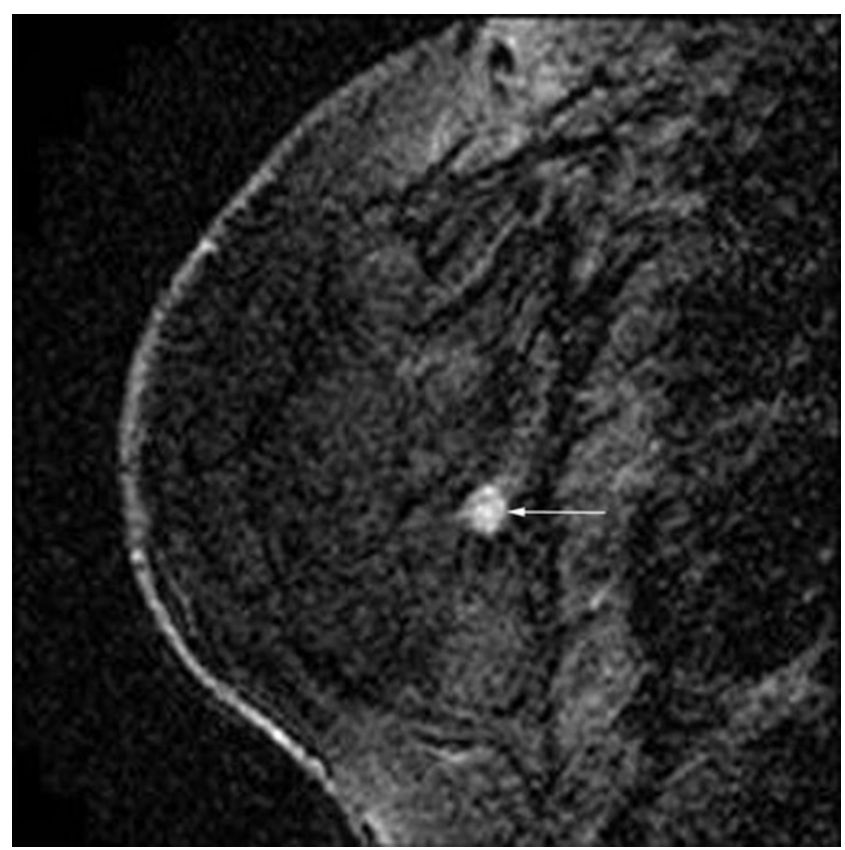

Fig. 11 A 57-year-old woman status post left mastectomy and latissimus dorsi myocutaneous flap reconstruction 12 years prior for diffuse DCIS. Enhanced T1W image demonstrates a 7-mm enhancing mass (arrow) in the posterior, lateral reconstructed left breast. This area was subsequently biopsied under ultrasound guidance with pathology demonstrating invasive carcinoma with ductal and lobular features involving fibro-adipose tissue and skeletal muscle breast parenchyma. However, an overlap exists between the two. In general, fibrosis can be differentiated from tumor by the degree and kinetics of contrast enhancement. Fibrosis is characterized by no enhancement or low-level enhancement that gradually increases with time (Fig. 8). Tumors exhibit more rapid and pronounced tissue enhancement, often with washout kinetics at delayed imaging [13]. However, falsepositive results in the early postoperative period and up to several months following radiation treatment are common due to the presence of enhancing granulation tissue [14].

\section{Fat necrosis}

Fat necrosis is a benign noninfectious inflammatory process of adipose tissue that is characterized histologically by necrotic fat cells with peripheral fibrosis and inflammatory cells. It is a common complication of TRAM flap reconstruction, with rates as high as $25 \%$ quoted in the literature. The cause is attributed to the marginally adequate blood supply in the periphery of the soft-tissue flap. Fat necrosis is often palpable, manifesting as a firm mass at physical examination, and the appearance can mimic that of tumor recurrence at both clinical evaluation and mammography.

At MR imaging, fresh fat necrosis is characterized by a round or irregular mass with central high-signal intensity on nonfat-suppressed unenhanced $\mathrm{T} 1 \mathrm{~W}$ images and high signal a

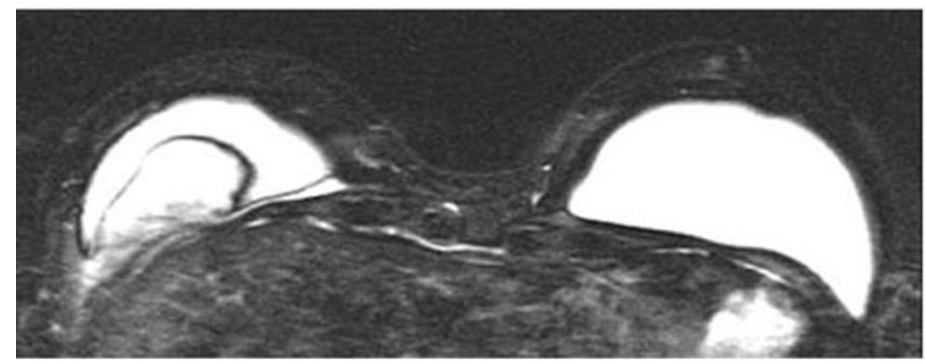

b

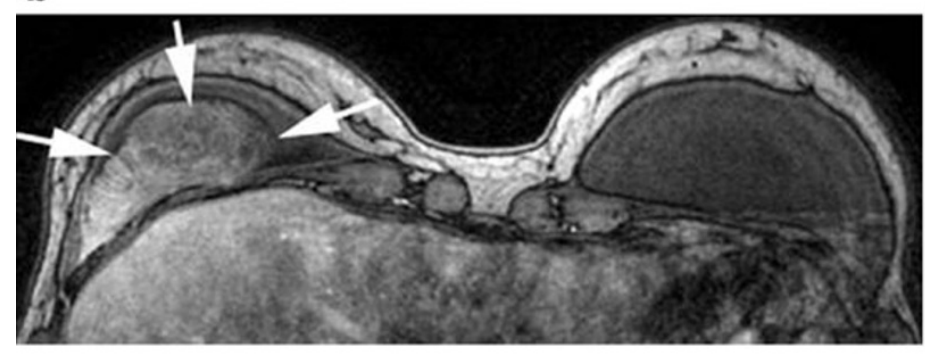

C

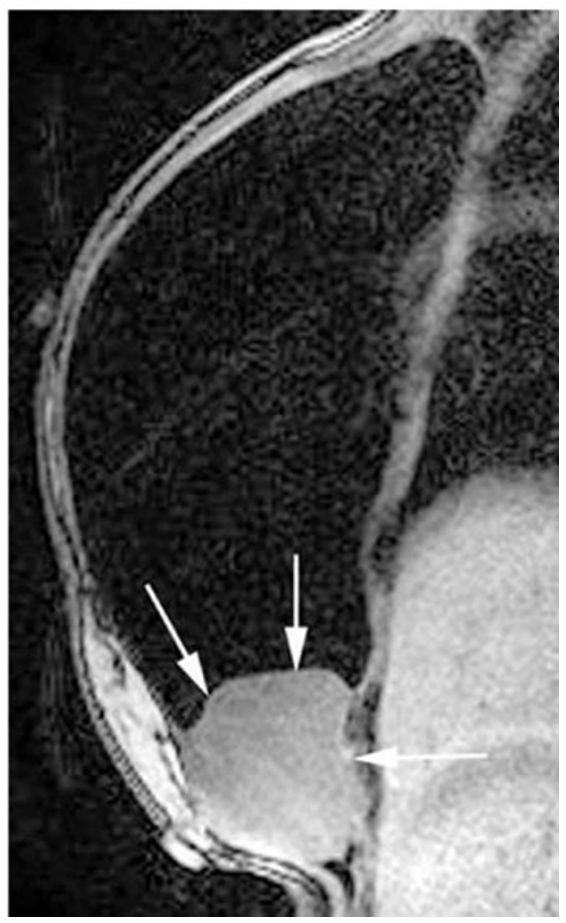

Fig. 12 A 46-year-old woman with implant reconstruction. Axial a unenhanced $\mathrm{T} 2 \mathrm{~W}$ fat-saturated, $\mathbf{b}$ enhanced $\mathrm{T} 1 \mathrm{~W}$, and $\mathbf{c}$ sagittal $\mathrm{T} 1$ silicone suppressed images show a circumscribed mass adjacent to the silicone implant in the inferior breast. The mass is bright on bright on $\mathrm{T} 2 \mathrm{~W}$ images and shows postcontrast enhancement. Biopsy proved this to be a spindle cell tumor 
on T2W images, and exhibits variable enhancement following administration of gadolinium contrast material. It could show fast initial enhancement and washout on the delayed enhancement images. Chronic fat necrosis or an oil cyst appears as a round mass with smooth margins, with central high signal intensity on nonfat-suppressed unenhanced T1W images and high signal on T2W images, and exhibits no enhancement on early or delayed images. It will follow the fat signal on all sequences [15]. The presence of central fat signal intensity is the key to differentiating fat necrosis from tumor recurrence, as breast cancers do not contain central fat (Fig. 9).

\section{Implant rupture}

If a saline breast implant ruptures, the implant deflates, causing the affected breast to change in shape and size (Fig. 10). The leaking saline is absorbed without complications. Patients usually undergo surgery and replacement of the implant. If silicone breast implants rupture, patients may remain asymptomatic or present with symptoms of breast pain, nodularity, or deformity. As we know, MRI is the most sensitive method for detection of intracapsular silicone implant rupture, and the linguine (collapsed and folded elastomer shell that is floating in gel) and keyhole (presence of silicone on both the inside and outside a radial fold) signs have been well documented in the literature. Macroscopic extrusion of silicone through the fibrous capsule into the surrounding parenchyma, pectoralis muscle, or lymph nodes may be seen as signs of extracapsular rupture.

\section{Malignant findings after flap reconstruction}

\section{Tumor recurrence}

Recurrence may occur locally within the reconstructed breast or in regional lymph nodes. At MR imaging, tumor recurrence following lumpectomy is characterized by a mass with low signal intensity on T1-weighted images, low to intermediate signal intensity on T2-weighted images, and avid and rapid enhancement following administration of gadolinium contrast material (Fig. 11). Other features, such as rim enhancement and spiculated morphology, increase the likelihood of malignancy. If there is an increase in the number or size of axillary or internal mammary lymph nodes, these should be viewed with suspicion for nodal recurrence. Axillary nodal recurrence can occur regardless of whether the initial sentinel node biopsy demonstrated metastatic tumor.

\section{De novo carcinoma}

Despite adequate surgical technique, residual glandular breast tissue can remain following mastectomy and breast reconstruction. An extremely extended time period between mastectomy and tumor appearance may indicate that a de novo cancer has formed. The distinction is important clinically, as a de novo ipsilateral breast cancer may be amenable to cure after local treatment, whereas local tumor recurrence following complete surgical excision is almost inevitably followed by the appearance of distant metastases. Other cancers may occur secondary to implant placement, such as mesenchymal tumors and sarcomas [19] (Fig. 12).

\section{References}

1. Stump A, Holton LH 3rd, Connor J, Harper JR, Slezak S, Silverman RP (2009) The use of acellular dermal matrix to prevent capsule formation around implants in a primate model. Plast Reconstr Surg 124:82-91

2. Delay E, Gounot N, Bouillot A, Zlatoff P, Rivoire M (1998) Autologous latissimus breast reconstruction: a 3-year clinical experience with 100 patients. Plast Reconstr Surg 102:14611478

3. Abdalla HM, Shalaan MA, Fouad FA, Elsayed AA (2006) Immediate breast reconstruction with expander assisted latissimus dorsi flap after skin sparing mastectomy. J Egypt Natl Canc Inst 18:134140

4. Hartrampf CR, Scheflan M, Black PW (1982) Breast reconstruction with a transverse abdominal island flap. Plast Reconstr Surg 69:216-225

5. Kanchwala SK, Bucky LP (2008) Optimizing pedicled transverse rectus abdominis muscle flap breast reconstruction. Cancer $\mathrm{J}$ $14: 236-240$

6. Granzow JW, Levine JL, Chiu ES, Allen RJ (2006) Breast reconstruction with gluteal artery perforator flaps. J Plast Reconstr Aesthet Surg 59:614-621

7. Granzow JW, Levine JL, Chiu ES, Allen RJ (2006) Breast reconstruction using perforator flaps. J Surg Oncol 94:441-454

8. Granzow JW, Levine JL, Chiu ES, LoTempio MM, Allen RJ (2007) Breast reconstruction with perforator flaps. Plast Reconstr Surg 120:1-12

9. Guerra AB, Metzinger SE, Bidros RS, Gill PS, Dupin CL, Allen RJ (2004) Breast reconstruction with gluteal artery perforator (GAP) flaps: a critical analysis of 142 cases. Ann Plast Surg 52:118-125

10. Kinkel K, Hylton NM (2001) Challenges to interpretation of breast MRI. J Magn Reson Imaging 13:821-829

11. Devon RK, Rosen MA, Mies C, Orel SG (2004) Breast reconstruction with a transverse rectus abdominis myocutaneous flap: spectrum of normal and abnormal MR imaging findings. Radiographics 24:1287-1299

12. Masser MR, Di Meo L, Hobby JA (1989) Tattooing in reconstruction of the nipple and areola: a new method. Plast Reconstr Surg 84:677-681

13. Dao TH, Rahmouni A, Campana F, Laurent M, Asselain B, Fourquet A (1993) Tumor recurrence versus fibrosis in the irradiated breast: differentiation with dynamic gadolinium-enhanced MR imaging. Radiology 187:751-755

14. Harms SE, Flamig DP, Hesley KL et al (1993) MR imaging of the breast with rotating delivery of excitation off resonance: clinical experience with pathologic correlation. Radiology 187:493-501

15. Kuhl C (2007) The current status of breast MR imaging. Part I. Choice of technique, image interpretation, diagnostic 
accuracy, and transfer to clinical practice. Radiology 244:356378

16. Losken A, Nicholas CS, Pinell XA, Carlson GW (2010) Outcomes evaluation following bilateral breast reconstruction using latissimus dorsi myocutaneous flaps. Ann Plast Surg 65:17-22

17. Strock LL (2009) Two-stage expander implant reconstruction: recent experience. Plast Reconstr Surg 124:1429-3618
18. Paterson P, Sterne GD, Fatah F (2006) Mesh assisted direct closure of bilateral TRAM flapdonor sites. J Plast Reconstr Aesthet Surg 59:347-351

19. Balzer BL, Weiss SW (2009) Do biomaterials cause implantassociated mesenchymal tumors of the breast? Analysis of 8 new cases and review of the literature. Hum Pathol 40(11):1564-1570, Epub 2009 Jun 21 